\title{
Ductal carcinoma in situ of the breast: immune cell composition according to subtype
}

\author{
Marie Colombe Agahozo ${ }^{1}$ Mieke R. van Bockstal ${ }^{1}{ }^{1}$ - Floris H. Groenendijk ${ }^{1}$ Thierry P. P. van den Bosch ${ }^{1}$. \\ Pieter J. Westenend ${ }^{2} \cdot$ Carolien H. M. van Deurzen ${ }^{1}$
}

Received: 8 March 2019 / Revised: 3 July 2019 / Accepted: 3 July 2019 / Published online: 2 August 2019

(c) United States \& Canadian Academy of Pathology 2019

\begin{abstract}
Ductal carcinoma in situ of the breast includes several subtypes with a divergent biological behavior. Data regarding the composition of ductal carcinoma in situ-associated immune cells and their potential role in progression is limited. We studied ductal carcinoma in situ-associated immune response by characterizing immune cell subsets according to ductal carcinoma in situ subtypes. Ductal carcinoma in situ-associated tumor infiltrating lymphocyte (TIL) density was evaluated based on hematoxylin and eosin (H\&E)-stained sections from 473 patients. Cases were subtyped based on ER, PR, and HER2. Patients were categorized as TIL-high or low. Ductal carcinoma in situ-associated immune cells of TIL-high cases were immunostained on whole slides with CD4, CD8, CD20, CD68, FOXP3, and PD-L1 (SP142 and SP263). In total, 131/473 patients (28.0\%) were considered as TIL-high. The percentage of TIL-high cases was significantly higher in HER2 + and triple-negative ductal carcinoma in situ $(P<0.0001)$. Overall, no statistical difference in immune cell composition according to subtypes was found. However, individual subtype comparison showed that ER + HER2 + cases had a significantly higher proportion of CD8 $+\mathrm{T}$ cells compared with triple-negative cases $(P=0.047)$. In TIL-high cases, PD-L1-SP142 expression on tumor cells was associated with subtype $(P=0.037)$; the lowest number of positive cases was observed in the HER2 + subtype (independent of ER). However, in TIL-high ductal carcinoma in situ, PD-L1 expression by both clones was limited. In conclusion, high numbers of TILs are predominantly observed in HER + and triple negative ductal carcinoma in situ. The ER + HER2 + subtype seems to attract a higher proportion of CD8 + T cells compared with the triple negative subtype. Among TIL-high cases, the HER2 + subgroup had the lowest PD-L1-SP142 expression on tumor cells. This suggests a more pronounced antitumor immunity in HER2+ ductal carcinoma in situ, which could play a role in its biological behavior.
\end{abstract}

\section{Introduction}

Invasive breast cancer is a heterogeneous disease that can be divided in several molecular subtypes with distinct clinical outcome [1]. Each molecular subtype has an immunohistochemical surrogate: luminal (ER+ with or without

Supplementary information The online version of this article (https:// doi.org/10.1038/s41379-019-0331-8) contains supplementary material, which is available to authorized users.

Marie Colombe Agahozo

m.agahozo@erasmusmc.nl

1 Department of Pathology, Erasmus MC Cancer Institute, Rotterdam, The Netherlands

2 Laboratory for Pathology, Dordrecht, The Netherlands
HER2+), basal (ER-, PR-, and HER2-, known as triple negative), and HER2-driven (ER-, HER2+) [2]. Invasive breast cancer subtypes trigger the immune system differently; high numbers of tumor infiltrating lymphocytes (TILs) are associated with triple-negative and HER2+ invasive breast cancer [3, 4]. Numerous reports illustrate a prognostic effect of TILs in invasive breast cancer [5-7].

Ductal carcinoma in situ is a nonobligate precursor of invasive breast cancer $[8,9]$. The detection rate of ductal carcinoma in situ has exponentially increased with the implementation of population screening by mammography [10-12]. Since the majority of patients are treated, data with respect to its treatment-naive behavior are limited. However, cases of regressive ductal carcinoma in situ have been described and an estimated $40-50 \%$ remains in situ carcinoma when left untreated [13-18]. Consequently, a substantial proportion of patients with ductal carcinoma in situ 
might be overtreated, resulting in unnecessary morbidity and health care costs. On the other hand, although a substantial proportion of breast cancer patients are detected in an early in situ stage, the incidence and morbidity of invasive breast cancer remains high [11]. Novel therapeutic and preventive strategies are therefore needed in order to optimize early risk assessment and intervention. In recent years, immunotherapy is regarded as one of the most promising approaches in cancer therapy [19]. However, the failure rate is substantial, potentially due to treatment at late stages and due to incomplete understanding of interactions between cancer cells and immune cells [20, 21]. Increased knowledge regarding the role of the immune response in an early disease stage as ductal carcinoma in situ could have major clinical consequences, as it could contribute to future immune modulation and potential prevention of progression.

Ductal carcinoma in situ has a heterogeneous biological behavior [22]. In studies restricted to patients with pure ductal carcinoma in situ, the frequency of HER2 + cases is relatively high (22-76\%) compared with invasive breast cancer studies, which report HER2 positivity in about $11-23 \%$ of cases [18, 23-28]. In contrast, the frequency of triple negative ductal carcinoma in situ is relatively low in pure ductal carcinoma in situ studies (4-7.5\%) compared with invasive breast cancer studies, which report that $10-13 \%$ of all invasive breast cancers are triple negative [23-27]. In line with this, there is a high frequency of extensive ductal carcinoma in situ adjacent to HER2+ invasive breast cancer, whereas the ductal carcinoma in situ component in triple negative invasive breast cancer, if present, is rather limited [27, 29, 30]. These data suggest a different biological behavior according to ductal carcinoma in situ subtype: HER2 + cases seems to either remain in situ for a longer period of time or has a rapid in situ growth rate. Triple negative ductal carcinoma in situ on the other hand seems to have a relatively rapid progression to invasion. These differences could be related to characteristics of the tumor cells and/or the microenvironment, i.e., the ductal carcinoma in situ-associated immune response.

Tumor associated immune cells are aggregated in heterogeneous infiltrates, of which TILs make up the largest group [31]. Data regarding the composition and role of TILs in ductal carcinoma in situ are limited, although this is an emerging field of research [32]. High TIL infiltration has been associated with high grade, triple negative and/or HER2 + ductal carcinoma in situ, which is in line with invasive breast cancer studies [25, 33-35]. Further analyses according to the composition of the immune cells showed an association between high numbers of $\mathrm{CD} 8+\mathrm{T}$ cells, ductal carcinoma in situ regression, and low ipsilateral recurrence risk $[18,36,37]$. On the other hand, CD19/CD20+ B cells and CD68+Mac387+ macrophages were associated with a larger ductal carcinoma in situ diameter, high grade, presence of comedonecrosis, and shorter recurrence free survival $[36,38]$. In addition, two recent studies reported on PD-L1 expression in ductal carcinoma in situ [34, 39]. Hendry et al. reported PD-L1 (clone: SP263, Ventana) expression on $11 \%$ of ductal carcinoma in situ cells and $25 \%$ of ductal carcinoma in situ-associated TILs ( $n=138$ cases). On the other hand, Thompson et al. reported no PD-L1 (clone: 5H1, Abcam) expression in ductal carcinoma in situ cells, but $81 \%$ of ductal carcinoma in situ-associated TILs ( $n=23$ cases).

These studies described above have some limitations, including study size and the use of tissue micro arrays for analysis of ductal carcinoma in situ-associated immune cells. Larger series were restricted to the analysis of one immune cell subset (such as CD20 + B cells or CD8+ $\mathrm{T}$ cells) or did not include ER, PR, and HER2 status $[18,25,38]$. Therefore, a more complete knowledge on how different immune subsets are associated with ductal carcinoma in situ subtype is lacking. Based on the biological behavior of ductal carcinoma in situ subtypes, as described above, we hypothesize that there is an association between subtype and the composition of the immune infiltrate. The extensive growth pattern of HER2 + cases suggests a potential protective/antiinvasion effect of the immune cells. The biological behavior of triple negative ductal carcinoma in situ on the other hand, which is also associated with dense immune infiltrates like HER2+ ductal carcinoma in situ, rather suggests immune evasion. The objective of this study was therefore to characterize the presence and composition of ductal carcinoma in situ-associated TILs in a large series of patients, in order to allow subgroup analyses according to subtype.

\section{Patients and methods}

\section{Patient selection and clinical data collection}

For this retrospective study, we included patients with a primary diagnosis of ductal carcinoma in situ, diagnosed at the Erasmus Medical Center Cancer Institute Rotterdam or the Laboratory for Pathology Dordrecht between 2000 and 2016. Clinical data collection included age, type of surgery (breast-conserving surgery or mastectomy), and follow-up. Patients with bilateral ductal carcinoma in situ were included as two cases. Patients with an ipsilateral invasive breast cancer within 6 months after the initial diagnosis of ductal carcinoma in situ were excluded. Other exclusion criteria were microinvasion and insufficient tissue. Recurrences were defined as ipsilateral histologically proven ductal carcinoma in situ, invasive breast cancer or regional/distant breast cancer metastases without a contralateral invasive 
breast cancer, occurring $\geq 6$ months after the initial ductal carcinoma in situ diagnosis. For this study, we used encoded leftover patient material, and therefore did not need an informed consent or approval from an ethical committee according to the Code of Conduct of the Federation of Medical Scientific Societies in The Netherlands [40].

\section{Pathological assessment of DCIS characteristics}

According to Dutch guidelines, ductal carcinoma in situ lesions $<4 \mathrm{~cm}$ are embedded completely since 2005 . In lesions with a diameter $>4 \mathrm{~cm}$, at least ten blocks of the lesion are embedded in order to exclude an invasive component. All hematoxin and eosin (H\&E)-stained whole sections of excision specimen were centrally reviewed by two observers to assess histological grade (based on nuclear atypia, according to the WHO classification), the predominant growth pattern, presence of comedonecrosis, microcalcifications, and TILs. In case of a disagreement, consensus was reached. Density of TILs was semi-quantified as minimal/absent, mild, moderate, or severe. Ductal carcinoma in situ-associated stroma was defined as the stroma within $1 \mathrm{~mm}$ of the duct, as described by Toss et al. [41]. Cut-offs for TIL density were defined as $0-5,5-30,30-50$, and $>50 \%$ of the ductal carcinoma in situ-associated stroma occupied by TILs, respectively, adapted from Beguinot et al. [35] and illustrated in Fig. 1. Distribution of TILs was scored as focal, patchy, or diffuse, defined as $<10,10-50$, and $>50 \%$ of all ductal carcinoma in situ ducts surrounded by TILs, respectively. Based on the density and distribution, cases were classified as either TIL-high or TIL-low. TILlow ductal carcinoma in situ was defined as a low density of TILs $(<30 \%$ of the ductal carcinoma in situ-associated stroma occupied by TILs) or a focal TIL distribution. TILhigh DCIS was defined as a moderate or high TIL density ( $>30 \%$ of the ductal carcinoma in situ-associated stroma occupied by TILs) and a patchy or diffuse TIL distribution. Ductal carcinoma in situ subtypes were determined by ER, PR, and HER2 immunohistochemistry (Table 1). ER and PR were defined as positive when at least $10 \%$ of the tumor cell was positive, which is extracted from the Dutch guidelines for IBC scoring [42]. HER2 was scored according to international guidelines [43]. HER2 In situ hybridization was performed in cases with equivocal HER2 immunohistochemistry. Ductal carcinoma in situ was classified as $\mathrm{ER}+\mathrm{PR} \pm \mathrm{HER} 2-, \mathrm{ER}+\mathrm{PR} \pm \mathrm{HER} 2+, \mathrm{ER}$ $-\mathrm{PR}-\mathrm{HER} 2+$, or triple negative.

\section{Pathological assessment of ductal carcinoma in situ- associated TILs}

To determine ductal carcinoma in situ-associated immune cell composition, we immunostained the DCIS-associated
Fig. 1 H\&E images of ductal carcinoma in situ with absent/ minimal (A), mild (B), moderate $(\mathbf{C})$, or severe (D) TIL density ( $\times 10$ magnification)

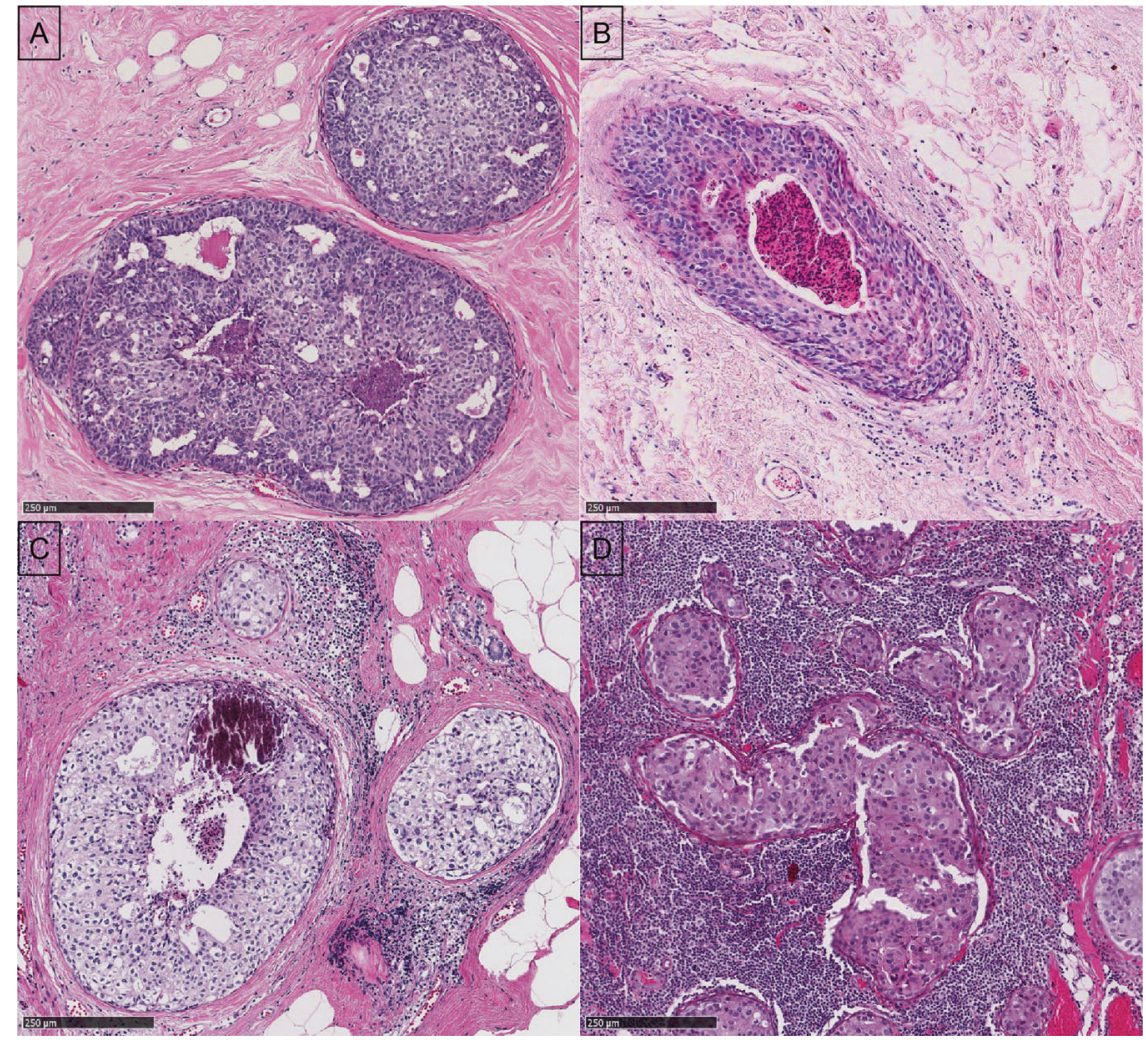


Table 1 Antibody characteristics and used protocol for whole tissue IHC

\begin{tabular}{|c|c|c|c|c|c|c|c|c|}
\hline Antibody* & Cell type & Type & Concentration & Company & Clone & Lot number & Procedure & Incubation time \\
\hline ER & n.a. & Anti-rabbit & $1 \mu \mathrm{g} / \mathrm{ml}$ & Ventana & SP1 & F02583 & Ultraview CC1 64' & $32 \mathrm{~min}$ \\
\hline PR & n.a. & Anti-rabbit & $1 \mu \mathrm{g} / \mathrm{ml}$ & Ventana & $1.00 \mathrm{E}+02$ & Y08684 & Ultraview CC1 36' & $12 \min$ \\
\hline HER2neu & n.a. & Anti-rabbit & $6 \mu \mathrm{g} / \mathrm{ml}$ & Ventana & $4 \mathrm{~B} 5$ & E06192 & Ultraview CC1 36' & $32 \mathrm{~min}$ \\
\hline CD4 & T-helper cell & Anti-rabbit & $2.5 \mu \mathrm{g} / \mathrm{ml}$ & Ventana & SP35 & G07304 & Ultraview CC1 64' & $8 \mathrm{~min}$ \\
\hline CD8 & Cytotoxic $\mathrm{T}$ cell & Anti-rabbit & $0.35 \mu \mathrm{g} / \mathrm{ml}$ & Ventana & SP57 & Y04591 & Optiview CC1 16' & $32 \mathrm{~min}$ \\
\hline CD20 & B cell & Anti-mouse & $0.3 \mu \mathrm{g} / \mathrm{ml}$ & Ventana & L2G & Y19660 & Ultraview CC1 64' & $44 \mathrm{~min}$ \\
\hline CD68 & Macrophage & Anti-mouse & $0.4 \mu \mathrm{g} / \mathrm{ml}$ & Ventana & KP1 & G01685 & Optiview CC1 16' & $8 \mathrm{~min}$ \\
\hline FOXP3 & Regulatory T-cell & Anti-mouse & $0.5 \mathrm{mg} / \mathrm{ml}$ & Thermofisher & 236A/E7 & 4339062 & Optiview CC1 $32^{\prime}$ & $32 \mathrm{~min}$ \\
\hline PDL1** & n.a. & Anti-rabbit & $7 \mu \mathrm{g} / \mathrm{ml}$ & Ventana & SP142 & Y03641 & Optiview CC1 64' & $16 \mathrm{~min}$ \\
\hline PDL1** & n.a. & Anti-rabbit & $1.61 \mu \mathrm{g} / \mathrm{ml}$ & Ventana & SP263 & Y2898U & Optiview CC1 $64^{\prime}$ & $16 \mathrm{~min}$ \\
\hline
\end{tabular}

*All antibodies were purchased from Ventana Roche, **PD-L1 antibodies were supplied by ROCHE

immune cells of TIL-high cases by automated immunohistochemistry using the Ventana Benchmark ULTRA (Ventana Medical System Inc.). Since TIL-low ductal carcinoma in situ has little to no TILs to characterize, characterization of TILs was restricted to TIL-high cases. Sequential 4- $\mu$ m-thick formal fixed paraffin-embedded whole tissue sections were stained for CD4 (T-helpers), CD8 (Cytotoxic T cells), CD20 (B cells), CD68, (Macrophages) and FOXP3 (Regulatory $\mathrm{T}$ cells). Table 1 provides an overview of used antibodies and protocols. In addition, PD-L1 (Programmed cell death ligand) was assessed using two clones (SP263 and SP142). Briefly, following deparaffinization and heat-induced antigen retrieval, tissue samples were incubated according to their optimized time and protocol with the antibody of interest. Incubation was followed by hematoxylin II counter stain for $8 \mathrm{~min}$ and then a blue coloring reagent for $8 \mathrm{~min}$ according to the manufacture's instructions (Ventana). The percentages of CD4+, CD8+, CD20+, and CD68+ immune cells were assessed manually by eyeballing, relatively to one another, with a combined score of $100 \%$. Next, the percentage of FOXP3 + cells was determined as a proportion of all immune cells. PDL1 expression in IBC is yet to be standardized; we therefore used two clones, which were scored individually. Membranous PD-L1 expression was scored for the ductal carcinoma in situ cells and the immune cells, as the percentage of positive cells. The cut-off for PD-L1 positivity was set at $1 \%$, both for ductal carcinoma in situ cells and immune cells. Two observers, blinded for ER, PR, and HER2 status, assessed all immunohistochemical staining.

\section{Statistical analysis}

IBM SPSS statistics 21.0 was used to perform statistical analysis. The Chi-square test was used to test for associations between clinicopathological characteristics, ductal carcinoma in situ subtypes based on immunohistochemistry, TILs, and PD-L1 expression. After testing for normal distribution, the Kruskal-Wallis test was used to analyze difference in the proportion immune subset density across the ductal carcinoma in situ subtypes. Differences in the proportion immune subsets between individual IHC-subtypes were analyzed using the Mann-Whitney $U$ test. Differences between PD-L1 expressions of both clones were assessed using the Wilcoxon Signed Ranks Test. A log-rank Mantel-Cox test was used to analyze the effect of TILs and PD-L1 expression on ipsilateral recurrence. Results were considered significant with a $P$-value $<0.05$.

\section{Results}

\section{General clinicopathologic characteristics}

In total, 501 cases were reviewed for this study. After central revision, 28 samples were excluded because of an ipsilateral IBC within 6 months $(n=22)$ or at deeper sectioning for additional immunohistochemical staining $(n=6)$. We therefore included 473 patients with pure ductal carcinoma in situ for further characterization of TILs. Table 2 provides an overview of clinicopathologic characteristics of this series. The median age at diagnosis was 58 years (range: 27-84), with a median follow-up time of 98 months (range: 24-218). Most patients $(62 \%)$ were treated with breast-conserving surgery. The majority of patients $(52 \%)$ were diagnosed with high-grade, and most cases were associated with calcifications (72\%). Ductal carcinoma in situ was subtyped as ER+PR $\pm \mathrm{HER} 2-(n=225 ; 54 \%), \mathrm{ER}+\mathrm{PR}$ $\pm \mathrm{HER} 2+(n=80 ; \quad 17 \%), \quad \mathrm{ER}-\mathrm{PR}-\mathrm{HER} 2+\quad(n=85$; $18 \%)$, TN $(n=22 ; 5 \%)$ or missing $(n=31)$. 
Table 2 Clinicopathological characteristics $(n=473)$

\begin{tabular}{|c|c|}
\hline \multicolumn{2}{|l|}{ Age at diagnosis (in years) } \\
\hline - Median (range) & $58(27-84)$ \\
\hline \multicolumn{2}{|l|}{ Surgery $(\%)$} \\
\hline - Breast-conserving surgery & $293(62)$ \\
\hline - Mastectomy & $180(38)$ \\
\hline \multicolumn{2}{|l|}{ Diameter (in cm) } \\
\hline - Median (range) & $2.00(0.1-23.0)$ \\
\hline - Missing $(n)$ & 65 \\
\hline \multicolumn{2}{|l|}{ Growth pattern $(\%)$} \\
\hline - Solid & $239(51)$ \\
\hline - Cribriform & $190(40)$ \\
\hline - Micropapillary & $37(8)$ \\
\hline - Papillary & $7(1)$ \\
\hline \multicolumn{2}{|l|}{ Grade (\%) } \\
\hline - Low & $63(13)$ \\
\hline - Intermediare & $166(35)$ \\
\hline - High & $244(52)$ \\
\hline \multicolumn{2}{|l|}{ Calcification (\%) } \\
\hline - Absent & $134(28)$ \\
\hline - Present & $339(72)$ \\
\hline \multicolumn{2}{|l|}{ Comedonecrosis (\%) } \\
\hline - Absent & $234(49)$ \\
\hline - Present & $239(51)$ \\
\hline \multicolumn{2}{|l|}{ TILs density (\%) } \\
\hline - Minimal & $212(45)$ \\
\hline - Mild & $110(23)$ \\
\hline - Moderate & $113(24)$ \\
\hline - Severe & $38(8)$ \\
\hline \multicolumn{2}{|l|}{ TILs distribution (\%) } \\
\hline - Focal & $71(15)$ \\
\hline - Patchy & $133(28)$ \\
\hline - Diffuse & $57(12)$ \\
\hline - N.A. & $212(45)$ \\
\hline \multicolumn{2}{|l|}{ Immunohistochemical subtype (\%) } \\
\hline - ER+PR $\pm \mathrm{HER} 2-$ & $255(54)$ \\
\hline - ER+PR \pm HER2 + & $80(17)$ \\
\hline - ER-PR-HER2+ & $85(18)$ \\
\hline - ER-PR-HER2- & $22(5)$ \\
\hline - Missing & $31(7)$ \\
\hline \multicolumn{2}{|l|}{ Ipsilateral recurrence $(\%)$} \\
\hline - None & $450(95)$ \\
\hline - Ductal carcinoma in situ & $4(1)$ \\
\hline - Invasive breast cancer & $5(1)$ \\
\hline - Invasive breast cancer and metastasis & $5(1)$ \\
\hline - Metastasis & $2(0)$ \\
\hline - Missing & $7(2)$ \\
\hline
\end{tabular}

\section{Presence of TILs according to ductal carcinoma in situ characteristics}

After stratification according to the TIL density and distribution, 131 out of 473 patients (28\%) were classified as TIL-high. TIL density and distribution were strongly associated $(P<0.0001, \quad$ Chi-square test, Supplementary Table 1).
TIL-high ductal carcinoma in situ was associated with high grade $(P<0.0001)$, a predominantly solid growth pattern $(P=0.006)$, presence of comedonecrosis $(P<$ $0.0001)$, larger diameter $(P<0.0001)$, and subtype based on immunohistochemistry $(P<0.0001)$. In multivariate analysis, only the diameter, grade, and subtype remained significantly associated with TILs (Table 3).

Overall, the majority of the ER-PR-HER2+ and triplenegative subtype was TIL high $(61 \%$ and $64 \%$, respectively), whereas the opposite was true for the ER+PR +HER2- and ER+PR+HER2+ subtype $(11 \%$ and $39 \%$, respectively) (Fig. 2). Within the ER+HER2- and ER-PRHER 2+ subtypes, there was a significant association between the grade and presence of TILs; high-grade cases had significantly more TILs compared with low-grade cases $(P=0.001$ for ER + HER $2-$ ductal carcinoma in situ and $P=0.008$ for ER $-\mathrm{PR}-\mathrm{HER} 2+$ ductal carcinoma in situ).

\section{Immune cell composition}

Due to insufficient remaining tissue, 13 TIL-high cases were excluded for immune cell composition analysis. We therefore included 118 TIL-high cases to study CD4, CD8, CD20, CD68, FOXP3, and PD-L1 expression. Overall, the majority of the ductal carcinoma in situ-associated immune cells were $\mathrm{CD} 4+$, followed by $\mathrm{CD} 20+, \mathrm{CD} 8+$, and CD68+ with a median of $46.0 \%$ (range 4-80), 34.5\% (range 2-90), $10.0 \%$ (range 2-40), and $5.0 \%$ (range 1-74), respectively (Fig. 3). The median proportion of FOXP3 expression was $3.0 \%$ (range 0-20).

In these TIL-high cases, PD-L1 expression was observed in both ductal carcinoma in situ cells and immune cells (Fig. 4). In total, 9 out of 119 cases (8\%) were PD-L1SP142 positive in ductal carcinoma in situ cells and 55 out of 119 cases (46\%) were PD-L1-SP263 positive in ductal carcinoma in situ cells. Regarding TILs, 97 out of 119 cases (82\%) were PD-L1-SP142 positive and 112 out of 119 cases (94\%) were PD-L1-SP263 positive. However, the overall PD-L1 expression was limited in both components. The median \% of PD-L1 positive ductal carcinoma in situ cells was 0 for both SP142 and SP263 (SP142 range 0-6 and SP263 range 0-52). The median \% of PD-L1+ immune cells was $1.0 \%$ for both SP142 and SP263 (SP142 range 0-7 and SP263 range 0-10). However, the expression of the PD-L1-SP263 clone was higher compared with the PD-L1SP142 clone, for both ductal carcinoma in situ and immune cells $(P<0.0001)$.

\section{TIL composition according to ductal carcinoma in situ characteristics}

Overall, there was no statistical difference in the composition of immune cell subsets according to ductal carcinoma 
Table 3 Ductal carcinoma in situ characteristics according to TILs

\begin{tabular}{|c|c|c|c|c|}
\hline & \multicolumn{2}{|c|}{ Overall TILs in ductal carcinoma in situ } & \multirow{2}{*}{$\begin{array}{l}P \text {-value } \\
\text { univariate } \\
\text { analysis }\end{array}$} & \multirow{2}{*}{$\begin{array}{l}P \text {-value } \\
\text { multivariate } \\
\text { analysis }\end{array}$} \\
\hline & TILs low $(n=342)$ & TILs high $(n=131)$ & & \\
\hline \multicolumn{5}{|l|}{ Age at diagnosis (years) } \\
\hline - Median (range) & $58.00(29.00-84.00)$ & $57.00(25.00-82.00)$ & 0.366 & 0.660 \\
\hline \multicolumn{5}{|l|}{ Surgery } \\
\hline - Breast-conserving therapy & $222(76)$ & $71(24)$ & 0.032 & 0.513 \\
\hline - Mastectomy & $120(67)$ & $60(33)$ & & \\
\hline Diameter (missing $n=53$ ) (in $\mathrm{cm}$ ) & & & $<0.0001$ & 0.044 \\
\hline - Median (range) & $1.65(0.1-23.0)$ & $2.90(0.1-16.0)$ & & \\
\hline \multicolumn{5}{|l|}{ Growth pattern $(\%)$} \\
\hline - Solid & $160(67)$ & $79(33)$ & & \\
\hline - Cribriform & $153(81)$ & $37(19)$ & 0.006 & 0.350 \\
\hline - Micropapillary & $23(62)$ & $14(38)$ & & \\
\hline - Papillary & $6(86)$ & $1(14)$ & & \\
\hline \multicolumn{5}{|l|}{ Grade $(\%)$} \\
\hline - Low (G1) & $60(90)$ & $3(5)$ & & \\
\hline - Intermediate (G2) & $149(90)$ & $17(10)$ & $<0.0001$ & $<0.0001$ \\
\hline - High (G3) & $133(55)$ & $111(45)$ & & \\
\hline \multicolumn{5}{|l|}{ Calcification (\%) } \\
\hline - Absent & $104(78)$ & $30(22)$ & 0.105 & 0.796 \\
\hline - Present & $238(70)$ & $101(30)$ & & \\
\hline \multicolumn{5}{|l|}{ Comedonecrosis $(\%)$} \\
\hline - Absent & $191(82)$ & $43(18)$ & $<0.0001$ & 0.208 \\
\hline - Present & $151(63)$ & $88(37)$ & & \\
\hline \multicolumn{5}{|c|}{ Immunohistochemical subtype (missing $n=31$ ) (\%) } \\
\hline - ER+PR \pm HER2- & $226(89)$ & $29(11)$ & & \\
\hline$-\mathrm{ER}+\mathrm{PR} \pm \mathrm{HER} 2+$ & $49(61)$ & $31(39)$ & $<0.0001$ & $<0.0001$ \\
\hline - ER-PR-HER2+ & $33(39)$ & $52(61)$ & & \\
\hline - ER-PR-HER2- & $8(36)$ & $14(64)$ & & \\
\hline \multicolumn{5}{|l|}{ Ipsilateral recurrence } \\
\hline - No & $330(73)$ & $120(27)$ & 0.338 & 0.175 \\
\hline - Yes & $10(63)$ & $6(38)$ & & \\
\hline
\end{tabular}

Significant values $(P<0.05)$ are indicated as bold

in situ subtypes. However, since HER2+ and triplenegative cases have a distinct biological behavior, we performed subgroup analyses restricted to these subtypes. This showed a significantly higher proportion of CD8 $+\mathrm{T}$ cells in the ER+HER2+ subtype compared with the triple negative subtype, although the absolute difference was limited (14 versus $10 \% ; P$-value $=0.047)$. The ER-HER2 + subtype was associated with a higher proportion of CD4+ T cells compared with the triple-negative group, although significance was not reached $(P$-value $=0.061)$. In addition, among TIL-high ductal carcinoma in situ, PD-L1-SP142 expression on tumor cells was associated with immunohistochemical subtype $(P=0.037)$; the lowest number of positive cases was observed in the HER2+ subgroups (both ER+HER2+ and ER-HER2+). There was no significant association between PD-L1 status (positive versus negative), and other features of neither the ductal carcinoma in situ component nor other immune cell parameters.

\section{TILs and ipsilateral recurrence}

In total, follow-up was available for 466 cases. The median follow-up time was 98 months (range 24-218 months). We 
Fig. 2 The proportion of TILs (TIL-low versus high cases) according to ductal carcinoma in situ (DCIS) subtype. The $X$ axis shows the ductal carcinoma in situ subtypes and the $Y$-axis illustrates the proportion of patients

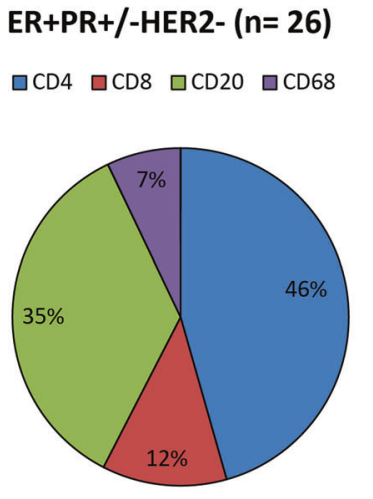

\section{$E R+P R+/-H E R 2+(n=27)$}

$\square \mathrm{CD} 4 \square \mathrm{CD} 8 \square \mathrm{CD} 20 \square \mathrm{CD} 68$

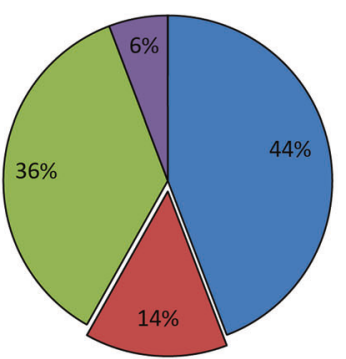

Overall TILs in DCIS

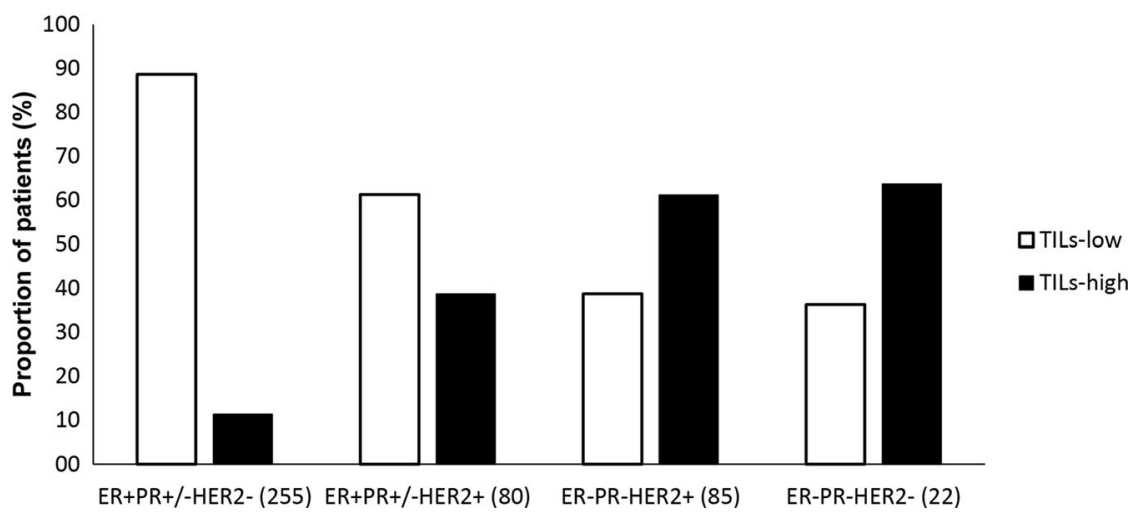

Fig. 3 The immune cell distribution according to subtype. The proportion of CD4 (blue), CD8 (red), CD20 (green), and CD68 (purple) expression is displayed as a percentage (\%) according to subtype

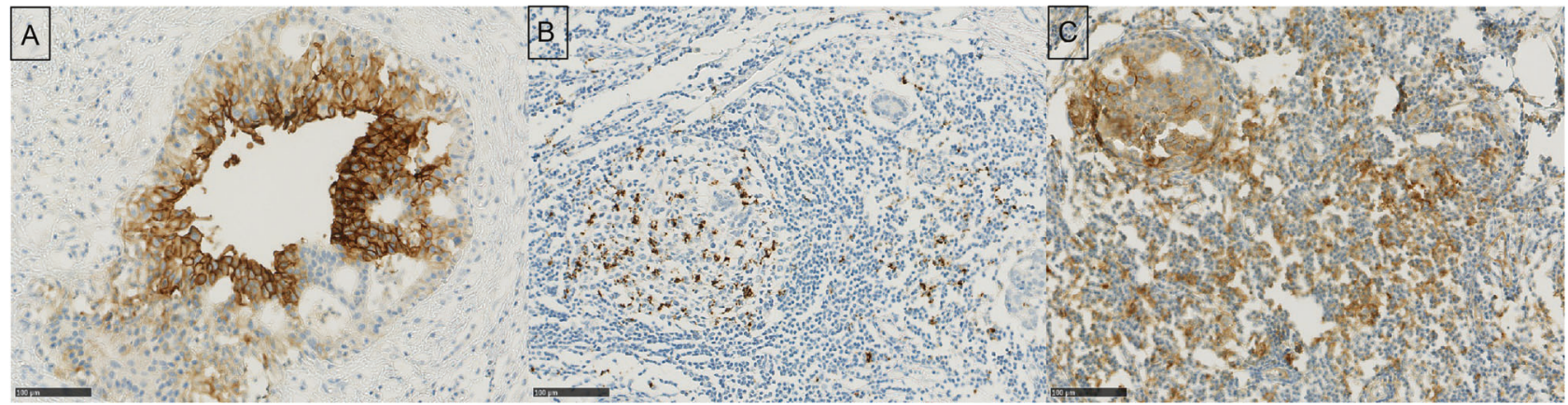

Fig. 4 Images of immunohistochemistry of PD-L1 expression in ductal carcinoma in situ cells (clone SP263) (A), ductal carcinoma in situassociated immune cells (clone SP142) (B) and in both (clone SP263) (C)

observed an ipsilateral recurrence in 16 patients (Table 2). The majority of these recurrences were invasive (10 out of 16); the remaining cases had a ductal carcinoma in situ recurrence. Half of the patients with an invasive recurrence (5 out of 10$)$ were associated with regional or distant metastasis. Two of these patients had a histologically proven distant breast cancer metastasis (liver and bone, respectively), in the absence of a proven primary invasive breast cancer. Overall, there were no significant associations between general ductal carcinoma in situ features and ipsilateral recurrence. The majority of patients with an ipsilateral recurrence had a primary diagnosis of $\mathrm{ER}+\mathrm{PR}$ +HER2- ductal carcinoma in situ $(n=10)$, remaining cases were HER2+. No recurrences were observed in those patients with a triple-negative ductal carcinoma in situ. In total, 6 out of 16 patients with a recurrence had TIL-high ductal carcinoma in situ in the original specimen; the remaining ten cases were TIL low. There was no significant 
association between TILs (TIL high versus low cases) and ipsilateral recurrence.

We compared the proportion of immune cell subsets and PD-L1 expression of TIL-high cases with ipsilateral recurrences to those without ipsilateral recurrence. There was no association between immune cell subset composition and ipsilateral recurrence. However, cases with an ipsilateral recurrence were associated with PD-L1-SP263 expression in ductal carcinoma in situ cells $(P=0.007)$, whereas there was no association between expression in TILs and recurrence $(P=0.522)$ by comparing PD-L1 positive versus negative cases (Supplementary table 2). Regarding PD-L1 expression as a continuous variable, however, expression levels in both ductal carcinoma in situ and TILs were significantly higher in the recurrent group versus the nonrecurrent group $(\mathrm{P}=0.002$ and $P=0.022$ respectively).

There was no significant association between PD-L1SP142 expression and ipsilateral recurrence $(P=0.408$ for ductal carcinoma in situ and $P=0.221$ for TILs) when comparing PD-L1 positive versus negative cases. However, the level of PD-L1-SP142 expression as a continuous variable in TILs was higher in the recurrent group compared with the nonrecurrent group $(P=0.045)$.

\section{Discussion}

This is the largest study evaluating the presence, including the composition, of ductal carcinoma in situ-associated immune cells in relation to ductal carcinoma in situ subtype based on immunohistochemistry. In our series, we found an association between ductal carcinoma in situ subtype and the presence of TILs, whereby ER-PR-HER2+ and triplenegative cases had the highest numbers of TILs, which is in line with previous studies [25, 33, 34].

The majority of ductal carcinoma in situ-associated immune cells were CD4+ T cells, of which $3 \%$ were likely to be regulatory $\mathrm{T}$ cells (FOXP3 positive). Overall PD-L1 expression was rather low in both ductal carcinoma in situ cells and immune cells. Nonetheless, high CD4+ FOXP3 +T-cell infiltrations, which partly express PD-L1, suggest a suppressed tumor immune microenvironment. This is in line with our finding, whereby PD-L1-SP263 expression was associated with an ipsilateral recurrence. Besides, this was also previously suggested by other studies demonstrating that $\mathrm{CD} 4+\mathrm{FOXP} 3+$ regulatory $\mathrm{T}$-cell infiltration was associated with large, highly proliferative ductal carcinoma in situ and microinvasion [35, 36, 39, 44].

Our data regarding ductal carcinoma in situ subtype and immune cell composition of TIL-high cases shows that the HER2 + subtype (independent of ER) had significantly less PD-L1-SP142 expression in tumor cells compared with the other subtypes, which suggests less immune suppression.
This is in line with a previous study of Thompson et al., reporting that HER2 + ductal carcinoma in situ did not have PD-L1-high TILs, whereas all included triple negative cases had PD-L1-high TILs [39]. Additionally, the ER+PR \pm HER2 + subtype was associated with a significantly higher proportion of $\mathrm{CD} 8+\mathrm{T}$ cells compared with the triple negative subtype. This association was also suggested by Morita et al., who reported an association between HER2+ ductal carcinoma in situ and high numbers of CD8 + T cells [18]. Previous studies also reported an association between high infiltration of ductal carcinoma in situ-associated CD8 $+\mathrm{T}$ cells and a low recurrence risk [36, 37], which is in line with invasive breast cancer studies [45-47]. These findings suggest a more pronounced anti-tumorigenic immune response in HER2+ ductal carcinoma in situ cases, which could play a role in its biological behavior.

To our knowledge, our study is the first to characterize ductal carcinoma in situ-associated immune cells and their association with subtype at this scale. Previous cohorts either evaluated immune cells, included ductal carcinoma in situ with microinvasion, small cohorts, did not consider the ER, PR, and HER2 status or they only evaluated TILs based on $\mathrm{H} \& \mathrm{E}$ in relation to $\mathrm{ER} / \mathrm{PR} / \mathrm{HER} 2$ status $[18,25,34-36,38,39,41,44]$. Moreover, we are the first to have used two sets of PD-L1 clones (SP142 and SP263, Ventana) to assess PD-L1 expression in TIL-high ductal carcinoma in situ. Several studies reported about the clinical results of immunotherapy in invasive breast cancer, but the nonresponse rate is still high [20,21]. There is currently no generally accepted PD-L1 clone or scoring system for invasive breast cancer or ductal carcinoma in situ. Increased knowledge regarding PD-L1 expression during breast carcinogenesis could contribute to future development of immune modulating therapies. Currently, immune modulation is mainly used for late stages of disease. However, if side effects and costs decrease during future drug development, it is likely that immune modulation will also be considered for earlier disease stages. The limited expression of PD-L1 expression in our DCIS cohort does however not support the use of immunotherapy in the in situ stage.

Nevertheless, our study also has some limitations. First, consensus for evaluation of TILs in ductal carcinoma in situ is yet to be reached, which results in different quantification methods, which restricts the comparison of results from several studies [41]. Besides, the quantification of TILs and immune cell subset composition is partly subjective, due to tissue selection bias and/or quantification methods, which could affect reproducibility between observers. We only analyzed PD-L1 expression of TIL-high cases, which limited our knowledge with respect to PD-L1 expression on tumor cells of TIL-low ductal carcinoma in situ. Besides, there are several limitations of the PD-L1 clones. The use of the SP142 antibody using the Ventana assay to assess 
PD-L1 expression was reported to be less sensitive than other commercially available antibodies, even though this antibody itself is equally sensitive [48]. This difference in sensitivity due to the assay could have impacted our results. In addition, we started with a large series, but the numbers of ipsilateral recurrences were low during a relatively limited follow-up time, which restricted the correlation of several pathological features with outcome.

In conclusion, high numbers of TILs are mainly observed in HER + and triple-negative ductal carcinoma in situ and the majority of these are CD4+ T cells. The ER+HER2+ subtype seems to attract a higher proportion of CD8+ $\mathrm{T}$ cells compared with the triple-negative subgroup. In addition, the TIL-high HER2 + subgroup (independent of ER) had the lowest PD-L1-SP142 expression on tumor cells. This suggests a more pronounced antitumorigenic immune response in HER2-positive ductal carcinoma in situ, which might play a role in its distinct biological behavior.

Acknowledgements This research was supported by Roche.

\section{Compliance with ethical standards}

Conflict of interest The authors declare that they have no conflict of interest.

Publisher's note: Springer Nature remains neutral with regard to jurisdictional claims in published maps and institutional affiliations.

\section{References}

1. Perou CM, Sùrlie T, Eisen MB, Rijn MVanDe, Jeffrey SS, Rees CA, et al. Molecular portraits breast cancer. Nature. 2000;533: 747-52.

2. Goldhirsch A, Winer EP, Coates AS, Gelber RD, Piccart-Gebhart M, Thürlimann B, et al. Personalizing the treatment of women with early breast cancer: highlights of the st gallen international expert consensus on the primary therapy of early breast Cancer 2013. Ann Oncol. 2013;24:2206-23.

3. Savas P, Salgado R, Denkert C, Sotiriou C, Darcy PK, Smyth MJ, et al. Clinical relevance of host immunity in breast cancer: from TILs to the clinic. Nat Rev Clin Oncol. 2016;13:228-41.

4. Kotoula V, Chatzopoulos K, Lakis S, Alexopoulou Z, Timotheadou E, Zagouri F, et al. Tumors with high-density tumor infiltrating lymphocytes constitute a favorable entity in breast cancer: a pooled analysis of four prospective adjuvant trials. Oncotarget. 2016;7:5074-87.

5. Aaltomaa S, Lipponen P, Eskelinen M, Kosma V, Marin S, Alhava E, et al. Lymphocyte Infiltrates as a prognostic variable in female breast cancer. Eur J Cancer. 1992;28:859-64.

6. Bense RD, Sotiriou C, Piccart-Gebhart MJ, Haanen JBAG, van Vugt MATM, de Vries EGE, et al. Relevance of tumor-infiltrating immune cell composition and functionality for disease outcome in breast cancer. J Natl Cancer Inst. 2017;109:djw192.

7. Mao Y, Qu Q, Chen X, Huang O, Wu J, Shen K. The prognostic value of tumor-infiltrating lymphocytes in breast cancer: a systematic review and meta-analysis. PLoS ONE. 2016;11: $\mathrm{e} 0152500$.
8. Kaur H, Mao S, Shah S, Gorski DH, Krawetz SA, Sloane BF, et al. Next-generation sequencing: a powerful tool for the discovery of molecular markers in breast ductal carcinoma in situ. Expert Rev Mol Diagn. 2013;13:151-65.

9. Lopez-Garcia MA, Geyer FC, Lacroix-Triki M, Marchió C, ReisFilho JS. Breast cancer precursors revisited: molecular features and progression pathways. Histopathology. 2010;57:171-92.

10. Barnes NLP, Ooi JL, Yarnold JR, Bundred NJ. Ductal carcinoma in situ of the breast How does DCIS develop? BMJ. 2012;344: e797.

11. Bleyer A, Welch G. Effect of three decades of screening mammography on breast-cancer incidence. N Engl J Med. 2012;367: 1998-2005.

12. Virnig BA, Wang S-Y, Shamilyan T, Kane RL, Tuttle TM. Ductal carcinoma in situ: risk factors and impact of screening. J Natl Cancer Inst Monogr. 2010;2010:113-6.

13. Collins LC, Tamimi RM, Baer HJ, Connolly JL, Colditz GA, Schnitt SJ. Outcome of patients with ductal carcinoma in situ untreated after diagnostic biopsy. Cancer. 2005;103:1778-84.

14. Page D, Dupont WD, Rogers LW, Jensen RA, Schuyler PA. Continued local recurrence of carcinoma 15-25 years after a diagnosis of low grade ductal carcinoma in situ of the breast treated only by biopsy. Cancer. 1995;76:1197-200.

15. Sanders ME, Schuyler PA, Dupont WD, Page DL. The natural history of low-grade ductal carcinoma in situ of the breast in women treated by biopsy only revealed over 30 years of long-term follow-up. Cancer. 2005;103:2481-4.

16. Chivukula M, Domfeh A, Carter G, Tseng G, Dabbs DJ. Characterization of high-grade ductal carcinoma in situ with and without regressive changes: diagnostic and biologic implications. Appl Immunohistochem Mol Morphol. 2009;17:495-9.

17. Wasserman JK, Parra-Herran C. Regressive change in high-grade ductal carcinoma in situ of the breast: histopathologic spectrum and biologic importance. Am J Clin Pathol. 2015;144:503-10.

18. Morita M, Yamaguchi R, Tanaka M, Tse GM, Yamaguchi M, Kanomata N, et al. CD8+ tumor-infiltrating lymphocytes contribute to spontaneous "healing" in HER2-positive ductal carcinoma in situ. Cancer Med. 2016;5:1-12.

19. Yang Y. Cancer immunotherapy: harnessing the immune system to battle cancer. J Clin Investig. 2015;125:3335-7.

20. Vonderheide RH, Domchek SM, Clark AS. Immunotherapy for breast cancer: what are we missing? Clin Cancer Res. 2017;23:2640-6. https://doi.org/10.1158/1078-0432.CCR-16-2569.

21. Hammerl D, Smid M, Timmermans A, Sleijfer S, Martens JW, Debets R. Breast cancer genomics and immuno-oncological markers to guide immune therapies. Semin Cancer Biol. 2018;52:178-88.

22. Gorringe KL, Fox SB. Ductal carcinoma in situ biology, biomarkers, and diagnosis. Front Oncol. 2017;7:248.

23. Zhou W, Jirström K, Amini R-M, Fjällskog M-L, Sollie T, Lindman $\mathrm{H}$, et al. Identification of a basal-like subtype of breast ductal carcinoma in situ. BMC Cancer. 2012;512:1-9.

24. Clark S, Warwick J, Carpenter R, Bowen R, Duffy S, Jones J. Molecular subtyping of DCIS: heterogeneity of breast cancer reflected in pre-invasive disease. Br J Cancer. 2010;104:120-7.

25. Pruneri G, Lazzeroni M, Bagnardi V, Tiburzio GB, Rotmensz N, DeCensi A, et al. The prevalence and clinical relevance of tumorinfiltrating lymphocytes (TILs) in ductal carcinoma in situ of the breast. Ann Oncol. 2017;28:321-8.

26. Parise CA, Caggiano V. Breast cancer survival defined by the ER/ PR/HER2 subtypes and a surrogate classification according to tumor grade and immunohistochemical biomarkers. J Cancer Epidemiol. 2014;2014:469251.

27. Doebar SC, van den Broek EC, Koppert LB, Jager A, Baaijens MHA, Obdeijn IMAM. et al. Extent of ductal carcinoma in situ according to breast cancer subtypes: a population-based cohort study. Breast Cancer Res Treat. 2016;158:179-87. 
28. Onitilo AA, Engel JM, Greenlee RT, Mukesh BN. Breast cancer subtypes based on ER/PR and Her2 expression: comparison of clinicopathologic features and survival. Clin Med Res. 2009;7:4-13.

29. Tot T. Early $(<10 \mathrm{~mm})$ HER2-positive invasive breast carcinomas are associated with extensive diffuse high-grade DCIS: implications for preoperative mapping, extent of surgical intervention, and disease-free survival. Ann Surg Oncol. 2015;22:2532-9.

30. Liao N, Zhang G-C, Liu Y-H, Li X-R, Yao M, Xu F-P, et al. HER2-positive status is an independent predictor for coexisting invasion of ductal carcinoma in situ of the breast presenting extensive DCIS component. Pathol Pr. 2010;207:1-7.

31. Burugu S, Asleh-Aburaya K, Nielsen TO. Immune infiltrates in the breast cancer microenvironment: detection, characterization and clinical implication. Breast Cancer. 2016;24:1-13.

32. Agahozo MC, Hammerl D, Debets R, Kok M, van Deurzen CHM. Tumor-infiltrating lymphocytes and ductal carcinoma in situ of the breast: friends or foes? Mod Pathol. 2018;31:1012-25.

33. Lee AHS, Happerfield LC, Bobrow LG, Millis RR. Angiogenesis and inflammation in ductal carcinomain situ of the breast. J Pathol. 1997;181:200-6.

34. Hendry S, Pang J-MB, Byrne DJ, Lakhani SR, Cummings MC, Campbell IG, et al. Relationship of the breast ductal carcinoma in situ immune microenvironment with clinico-pathological and genetic features. Clin Cancer Res. 2017;23:5210-7.

35. Beguinot M, Dauplat M-M, Kwiatkowski F, Lebouedec G, Tixier $\mathrm{L}$, Pomel C, et al. Analysis of tumour-infiltrating lymphocytes reveals two new biologically different subgroups of breast ductal carcinoma in situ. BMC Cancer. 2018;18:129.

36. Campbell MJ, Baehner F, O’Meara T, Ojukwu E, Han B, Mukhtar $\mathrm{R}$, et al. Characterizing the immune microenvironment in highrisk ductal carcinoma in situ of the breast. Breast Cancer Res Treat. 2017;161:17-28.

37. Semeraro M, Adam J, Stoll G, Louvet E, Chaba K, PoirierColame V, et al. The ratio of CD8 C /FOXP3 T lymphocytes infiltrating breast tissues predicts the relapse of ductal carcinoma in situ. Oncoimmunology. 2016;5:2162-402x.

38. Miligy I, Mohan P, Gaber A, Aleskandarany MA, Nolan CC, Diez-Rodriguez $\mathrm{M}$, et al. Prognostic significance of tumour infiltrating B-lymphocytes in breast ductal carcinoma in situ. Histopathology. 2017;71:258-68.

39. Thompson E, Taube JM, Elwood H, Sharma R, Meeker A, Warzecha HN, et al. The immune microenvironment of breast ductal carcinoma in situ. Mod Pathol. 2016;29:249-58.

40. FEDERA. Human tissue and medical research: code of conduct for responsible use (2011). Rotterdam 2011.

41. Toss MS, Miligy I, Al-Kawaz A, Alsleem M, Khout H, Rida PC, et al. Prognostic significance of tumor-infiltrating lymphocytes in ductal carcinoma in situ of the breast. Mod Pathol. 2018;31: 1226-36.

42. Dutch Institute for Clinical Auditing. Factsheet Indicatoren NABON Breast Cancer Audit (NBCA) 2017. Leiden. 2017.

43. Wolff AC, Hammond MEH, Hicks DG, Dowsett M, McShane $\mathrm{LM}$, Allison $\mathrm{KH}$, et al. Recommendations for human epidermal growth factor receptor 2 testing in breast cancer: American Society of Clinical Oncology/College of American Pathologists clinical practice guideline update. J Clin Oncol. 2013;31: 3997-4013.

44. Abba MC, Gong T, Lu Y, Lee J, Zhong Y, Lacunza E, et al. A molecular portrait of high-grade ductal carcinoma in situ. Cancer Res Tumor Stem Cell Biol. 2016;18:3980-90.

45. Miyashita M, Sasano H, Tamaki K, Hirakawa H, Takahashi Y, Nakagawa $\mathrm{S}$, et al. Prognostic significance of tumor-infiltrating $\mathrm{CD} 8+$ and FOXP3 + lymphocytes in residual tumors and alterations in these parameters after neoadjuvant chemotherapy in triplenegative breast cancer: a retrospective multicenter study. Breast Cancer Res. 2015;17:124.

46. Matkowski R, Gisterek I, Halon A, Lacko A, Szewczyk K, Staszek U, et al. The prognostic role of tumor-infiltrating CD4 and CD8 T lymphocytes in breast cancer. Anticancer Res. 2009;29: 2445-51.

47. Seo AN, Lee HJ, Kim EJ, Kim HJ, Jang MH, Lee HE, et al. Tumour-infiltrating CD8+ lymphocytes as an independent predictive factor for pathological complete response to primary systemic therapy in breast cancer. Br J Cancer. 2013;109:2705-13.

48. Gaule P, Smithy JW, Toki M, Rehman J, Patell-Socha F, Cougot $\mathrm{D}$, et al. A quantitative comparison of antibodies to programmed cell death 1 Ligand 1. JAMA Oncol. 2017;3:256. 